INPLASY

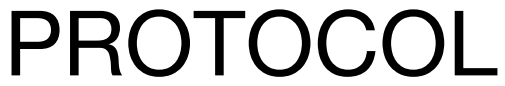

To cite: Meng et al. Tuina plus acupuncture for post-stroke depression: a protocol of a systematic review and metaanalysis. Inplasy protocol 202140098. doi:

10.37766/inplasy2021.4.0098

Received: 19 April 2021

Published: 19 April 2021

Corresponding author: Deyu Cong

cong_de_yu@sina.com

Author Affiliation:

Department of Acupuncture and Tuina, Changchun

University of Chinese

Medicine

Support: Grant number 2018YFC1706000.

Review Stage at time of this submission: The review has not yet started.

Conflicts of interest:

None declared.

\section{Tuina plus acupuncture for post- stroke depression: a protocol of a systematic review and meta-analysis}

Meng, M¹; Hu, G2; Yang, K3; Wang, H4 Han, Y5; Pan, T6; Lou, H7; Zhang, Y8; Wang, Y9; Cong, D10.

Review question / Objective: At present, there is no systematic review of Tuina combined with acupuncture treatment in the treatment of post-stroke depression, so this study will evaluate the efficacy and safety of Tuina combined with acupuncture in the treatment of post-stroke depression, and provide evidence for clinical decision-making of tuina and acupuncture.

Condition being studied: Stroke is the second leading global cause of death behind cardiovascular disease .It is also one of the diseases with high mortality and disability rate in the world. Post-stroke depression (PSD) is the most common mental health issue, afflicting around $33 \%$ of stroke survivors. PSD has a negative impact on the rehabilitation, recuperation of motor and cognitive deficits following stroke and significantly increases the chances of relapsing neurovascular events. The medical cost of patients with post-stroke depression is about four times as much as poststroke patients without depression not only severely restricts the ability of patients, reduces the quality of life, but also causes psychological impact on patients' rehabilitation, and brings a great burden to families and society.

INPLASY registration number: This protocol was registered with the International Platform of Registered Systematic Review and Meta-Analysis Protocols (INPLASY) on 19 April 2021 and was last updated on 19 April 2021 (registration number INPLASY202140098).

\section{INTRODUCTION}

Review question / Objective: At present, there is no systematic review of Tuina combined with acupuncture treatment in the treatment of post-stroke depression, so this study will evaluate the efficacy and safety of Tuina combined with acupuncture in the treatment of post-stroke depression, and provide evidence for clinical decisionmaking of tuina and acupuncture.

Condition being studied: Stroke is the second leading global cause of death 
behind cardiovascular disease .It is also one of the diseases with high mortality and disability rate in the world. Post-stroke depression (PSD) is the most common mental health issue, afflicting around 33\% of stroke survivors. PSD has a negative impact on the rehabilitation, recuperation of motor and cognitive deficits following stroke and significantly increases the chances of relapsing neurovascular events. The medical cost of patients with post-stroke depression is about four times as much as poststroke patients without depression not only severely restricts the ability of patients, reduces the quality of life, but also causes psychological impact on patients' rehabilitation, and brings a great burden to families and society.

\section{METHODS}

Participant or population: We will consider patients with a clinical diagnosis of PSD irrespective of their gender, age, severity, and disease duration.

Intervention: Intervention measures should be Tuina combined with acupuncture to treat post-stroke depression. If combined with other methods, only the control group with the same intervention measures as the experimental group will be included.

Comparator: The treatment group using tuina plus acupuncture, while the control group receives treatment of oral medication, Chinese herbal medication, physical therapy, botox injections and so on or even with no treatment will be included.

Study designs to be included: Randomized controlled trials (RCTs).

Eligibility criteria: This review will include randomized controlled trials (RCTs) on Tuina combined with acupuncture for PSD published in Chinese and English.

Information sources: The following electronic databases will be searched: PubMed, the Cochrane Library, EMBASE, Web of Science, Medline, China National Knowledge Infrastructure, Chinese
Biomedical Literature Database, China Science and Technology Journal Database, and Wan Fang databases. We will consider articles published between the database initiation and April 2021.

Main outcome(s): The primary outcomes will include the Hamilton depression rating scale (HDRS)and the effective rate.

Quality assessment / Risk of bias analysis: Two reviewers will separately assess the risk of bias of the selected RCTs using the Cochrane risk of bias assessment tool. This tool has the following 7 domains: random sequence generation, allocation concealment, blinding of participants and personnel,blinding of outcome assessment, incomplete outcome data, selective reporting, and other bias. A bias value of "low," "unclear," or "high" will be used to rank the risk of bias. These even domains will be separately appraised by 2 reviews and discrepancies will be addressed by consulting a third reviewer.

Strategy of data synthesis: If studies are adequately homogeneous in design and comparison, we will conduct data synthesis using Review Manager Software 5.4. The fixed-effects or random effects model will be chosen depending on the 12 value. A $95 \%$ confidence interval will be the effective size for data synthesis. We will perform qualitative analysis if the data is not fit for quantitative analysis.

Subgroup analysis: We will perform subgroup analysis among patient conditions, treatment methods, and outcome measurements if feasible.

Sensitivity analysis: In order to test the robustness of the main decisions in the review process, we will conduct a sensitivity analysis. The main analysis points include the impact of method quality, sample size, and missing data on the study. The meta-analysis will be reused, and more inferior quality studies will be excluded. The results will be compared and discussed according to the results.

Country(ies) involved: China. 
Keywords: Tuina, acupuncture, protocol, post-stroke depression, systematic review.

Contributions of each author:

Author 1 - Meng Meng.

Author 2 - Guanyu Hu.

Author 3 - Kang Yang.

Author 4 - Heran Wang.

Author 5 - Yiran Han.

Author 6 - Ting Pan.

Author 7 - Huijuan Lou.

Author 8 - Ye Zhang.

Author 9 - Yufeng Wang.

Author 10 - Deyu Cong. 